\title{
Research on Investor Sentiment in the IPO Stock Market
}$$
\text { Ziyu Liu }{ }^{1, a} \text {, Han Yang }{ }^{2, b} \text {, Weidi Zhang }{ }^{3, \mathrm{c}} \text { and Xiaoli Hu }{ }^{\text {4, d }}
$$ \\ ${ }^{1}$ Science and Engineering Department, Communication University of China, Beijing, China; \\ ${ }^{2}$ Science and Engineering Department, Communication University of China, Beijing, China; \\ ${ }^{3}$ Science and Engineering Department, Communication University of China, Beijing, China; \\ ${ }^{4}$ Science and Engineering Department, Communication University of China, Beijing, China.

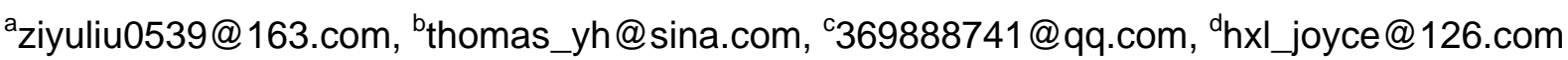

Keywords: Investor sentiment; IPO stock; Cumulative abnormal return; Cluster analysis; ANOVA

\begin{abstract}
The paper developed a sentiment factor to classify the window period into three sentiment levels (low, medium and high), discussing how the IPO stock performance was significantly affected by investor sentiment through analysis of variance, where the sentiment factor was proposed by means of cluster analysis towards three investor sentiment proxy indicators (turnover rate, number of new investors and consumer confidence index) and the stock performance data was consist of cumulative abnormal return, cumulative return and cumulative volatility. Conclusions are drawn that the IPO stock performance is basically not affected by market sentiment in the early stage, however, after a certain period the market sentiment gradually shows obvious effect on IPO stock performance, manifested by gradually being independent of the IPO market and conforming to the general market.
\end{abstract}

\section{Introduction}

As is known that the stock market is driven by emotion so investor sentiment is not always synonymous with fundamental value. Traders make money by finding stocks that are overvalued or undervalued based on market sentiment, or investor sentiment. Drawing on previous domestic and foreign scholars measuring investor sentiment, two mainly method are raised, the direct measurement using the ratio of bulls and bears and the indirect one adopting proxy indicators of investor sentiment through empirical and theoretical analysis.

This paper discusses the impact of investor sentiment on the Chinese IPO stock market. 344 stocks in all, listed on the Shanghai Stock Exchange in 2014 and 2015 are studied to find out how the IPO stock return is significantly affected by investor sentiment in the initial 150 days. Considering the information asymmetry and certain emotional release process are often existed in new listed stocks, we set an assumption that at the first phase stock return might fail to represent the market sentiment at first and begin corresponding to the characteristics of the market sentiment after a certain period.

Compared with existing researches, contributions of this paper are mainly reflected in the following areas:(1) selecting proxy indicators of investor sentiment appropriate to Chinese stock market and using hierarchical clustering and k-means clustering to classify the observation period, in which process an investor sentiment factor is set;(2) adopting the proposed sentiment factor in the analysis of variance to test the effectiveness of the 3-level factors on IPO stock return;(3) respectively discussing by the day and by the month whose conclusion supports and verifies each other.

We initially put forward that IPO stock performance is not affected significantly in the early stage (initial three months) while is affected significantly after a certain period (three months later).

\section{Data}

Investor Sentiment and Stock Performance. Investor sentiment, also called the "market sentiment", is the feeling or tone of a market, or its crowd psychology, as revealed through the activity and price movement of the securities traded in that market. It is generally described as bearish or bullish. 
Measurement of market sentiment has been lack of standards. To avoid the restriction of using single indicator to describe the stock market sentiment, we adopted the composite proxy indicators to represent. Combining the in the previous study and the situation of Chinese stock market, three proxy indicators (including turnover rate, number of new investors and consumer confidence index) are selected to characterize certain investors psychologies, which reflect the investor sentiment in a way, such as divergence, frequent trading behavior, overconfidence, etc. Plus, due to the lack of data (IPO was suspended in the Chinese market for nearly four months in 2015), IPO numbers and first-day trading price are not used.

In the study we chose 344 IPO stocks listed on the Shanghai Stock Exchange in 2014 and 2015 and collected their market data (including stock name, stock code, issue date, issue price, issue share, etc.) in the initial 150 days since the stock is listed. For each stock, cumulative abnormal return, cumulative return and cumulative volatility were calculated to represent the IPO stock performance. Formulas and Explanations. In this section we give definitions of the formulas and explanations for the indicators mentioned above. Investor sentiment proxy indicators refer to turnover rate, number of new investor accounts and consumer confidence index.

Turnover Rate. Turnover rate indicates the velocity with which stock of finished goods is sold, and is widely used to describe investor sentiment by scholars. Below shows its formula.

$\operatorname{TURN}_{j}=\mathrm{VOL}_{j} / O S_{j}$

where $T U R N_{j}$ refers to turnover rate of the Shanghai Stock Exchange, $V O L_{j}$ refers to trading volume, $O S_{j}$ refers to total outstanding, j refers to the trading day or trading month.

We collected daily turnover rate from the official website of the Shanghai Stock Exchange, as monthly turnover rate is calculated based on the daily data.

Number of New Investors. Comparing with the mature capital market in the developed country, the number of new investors in Chinese stock market changes obviously, usually more in the bull market and less in the bear market, which reflects investor sentiment effectively. Shiller (2005) proposed that the formation of the bull market was due to the rapidly increasing investors involved directly in the stock market, providing a theoretical basis for using it as an investor sentiment proxy indicator, collecting from the website of CSDC (China Securities Deposition and Clearing).

Consumer Confidence Index (CCI). An index by the NBS (National Bureau of Statistics) that measures how optimistic or pessimistic consumers are with respect to the economy. The idea behind CCI is that if consumers are optimistic, they tend to purchase more goods and services (stocks in this case). This increase in spending inevitably stimulates the whole economy. According to the method adopted by Huang Xuhong (2012), we used the first-order difference of CCI as another proxy indicator to represent the investor sentiment.

Stock Performance Indicators. Stock performance proxy indicators describe the return and volatility of stocks, with formulas below.

$$
\begin{aligned}
& R_{i t}=\left(P_{i t}-P_{i 0}\right) / P_{i 0} \\
& V_{i t}=\operatorname{Var}\left(R_{i t}\right) \\
& C A R_{i t}=R_{i t}-R_{i m}
\end{aligned}
$$

where $R_{i t}$ refers to cumulative return, $R_{i m}$ refers to market return, $V_{i t}$ refers to cumulative volatility, $C A R_{i t}$ refers to cumulative abnormal return, $P_{i t}$ refers to the closing price at time t, $P_{i 0}$ refers to the closing price in the first day the stock listed, i refers to certain stock, $t$ refers to the period since the stock was listed. For simple description, we assume that each month has 30 calendar days and 20 trading days. Yet in the actual calculation, the analysis are depended on the factual data.

\section{Model}

Calculated out indicators representing stock performance and investor sentiment, we begin discussing about the interaction between the two categories. As the assumption we previously set, it is supposed that the IPO stock performance fails to reflect investor sentiment at first and gradually corresponds to the characteristics of market emotion after a period. 
Hierarchical Clustering and K-means Clustering. Hierarchical clustering and k-means clustering are common cluster analysis methods of classification. In the study, the two methods are combined to segment various investor sentiment levels, including the first step to determine the best cluster number and the second to classify them.

Hierarchical clustering is a method of cluster analysis which seeks to build a hierarchy of clusters. Set the cluster number from 2 to 4, it can be seen that the result was better when we divided the sentiment indicators into three clusters.

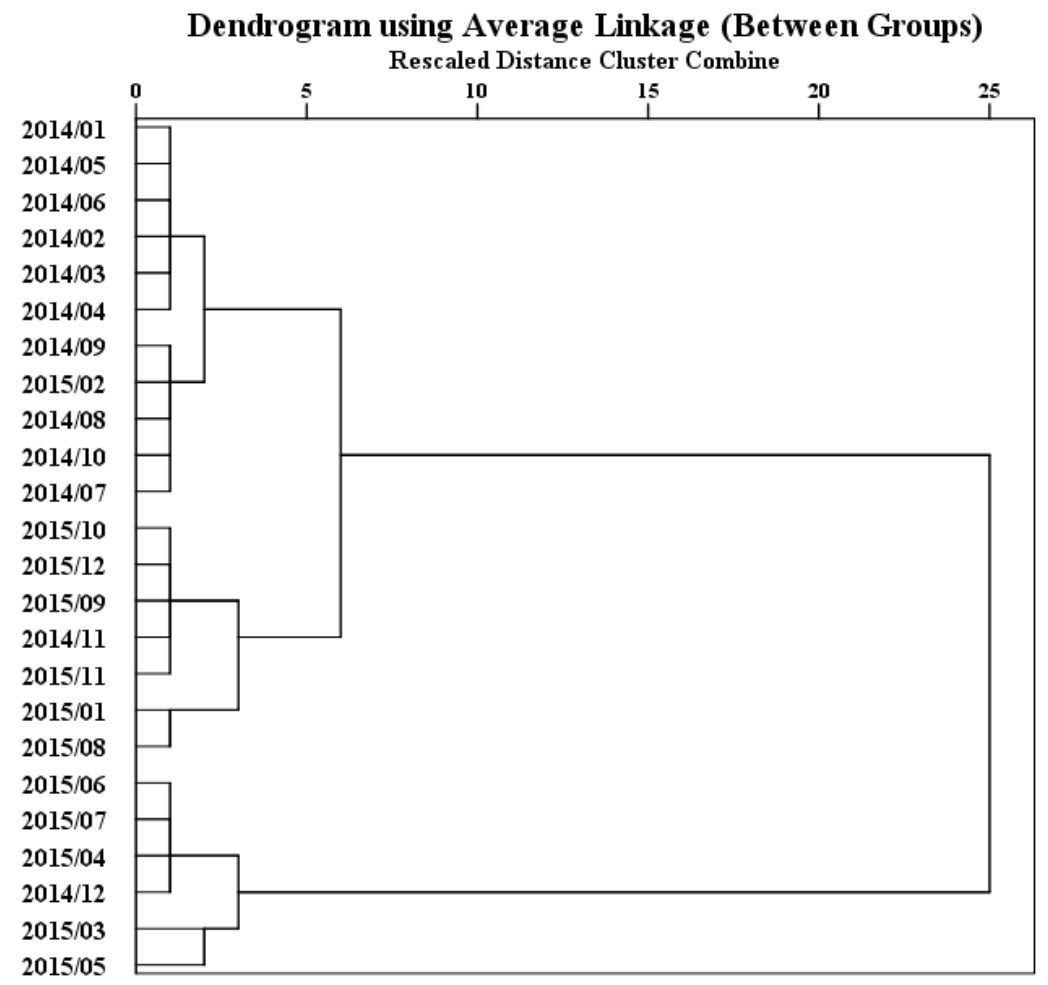

Fig. 1 Dendrogram of investor sentiment in 24 trading months by hierarchical clustering

K-means clustering, aiming to find the positions of the clusters that minimize the distance from the data points to the cluster, its main idea is to define k centroids, one for each cluster. Then, each trading day and trading month during the observation period can be classified into three investor sentiment levels (high, medium, low) respectively based on the three investor sentiment proxy indicators.

We proposed a sentiment factor to represent diverse investor sentiment levels and matched the factor with each stock's performance data both by the day and by the month.

$\operatorname{SENTI}_{j}=1,2,3$ (1: low 2: medium 3: high)

Where $\mathrm{j}$ refers to certain trading day or trading month.

Homogeneity of Variance Test. Levene's test is an inferential statistic used to assess the equality of variances for two or more groups. Some of the procedures, typically assuming homoscedasticity, include analysis of variance. If the p-value of Levene's test is less than 0.05 (the typical significance level), the obtained differences in sample variances are unlikely to have occurred, which is free from homoscedasticity assumptions. Thus, we standardize some stock performance indicators (those failed to meet the assumption) under each sentiment level to satisfy the requirement of analysis of variance. One-Way ANOVA. One-way analysis of variance (abbreviated one-way ANOVA), proposed by R.A.Fisher, uses the $\mathrm{F}$ distribution to compare means of three or more samples. It produces an F-statistic, the ratio of the variance calculated among the means (between groups) to the variance within the samples (within groups). A higher ratio therefore implies that the samples were drawn from populations with different mean values. To calculate the F-ratio:

$$
F_{k-1, N-k}=\frac{M S_{B}}{M S_{W}}=\frac{S S_{B} /(k-1)}{S S_{W} /(N-k)}
$$

Where $M S_{B}$ is the residual sum of squares between groups, $M S_{W}$ is that within groups. Under the null hypothesis, $\mathrm{F}$ have an $\mathrm{F}$ distribution, with $(\mathrm{k}-1, \mathrm{n}-\mathrm{k})$ degrees of freedom. 


\section{Empirical Analysis}

We selected 344 stocks listed on the Shanghai Stock Exchange in 2014 and 2015 to calculate out stock performance proxy indicators, then collected investor sentiment proxy indicators during the period and classify them into three sentiment levels by means of cluster analysis, finally, analysis of variances was adopted to find out the impact of investor sentiment on stock performance.

The clustering procedure gave a SENTI label to 490 trading days during 730 calendar days (or 24 calendar months) in 2014 and 2015 so that each stock performance data (time series data) was matched with a certain investor sentiment level. Subsequently, homogeneity of variance test and ANOVA were implemented, standardization included. Below are the results of monthly data.

Table 1 Result of homogeneity of variance test and ANOVA

\begin{tabular}{|c|c|c|c|c|c|}
\hline \multirow{2}{*}{$\begin{array}{c}\begin{array}{c}\text { Sentiment } \\
\text { Factor }\end{array} \\
\text { SENTI } \\
(0,20)\end{array}$} & \multirow{2}{*}{$\begin{array}{c}\text { Stock } \\
\text { Performance }\end{array}$} & \multicolumn{2}{|c|}{$\begin{array}{c}\text { Test of } \\
\text { Homogeneity of } \\
\text { Variance }\end{array}$} & \multicolumn{2}{|c|}{ ANOVA } \\
\hline & & 0.129 & accept & 1 & accept \\
\hline & V1 & 0.913 & accept & 1 & accept \\
\hline \multirow[t]{2}{*}{$(20,40)$} & R2 & 0.151 & accept & 0.055 & accept \\
\hline & V2 & 0.151 & accept & 0.616 & accept \\
\hline \multirow[t]{2}{*}{$(40,60)$} & R3 & 0.192 & accept & 1 & accept \\
\hline & V3 & 0.6 & accept & 1 & accept \\
\hline \multirow[t]{2}{*}{$(60,80)$} & R4 & 0.069 & accept & $0^{*}$ & reject \\
\hline & V4 & 0.206 & accept & 1 & accept \\
\hline \multirow[t]{2}{*}{$(80,100)$} & R5 & 0.295 & accept & $0.033 *$ & reject \\
\hline & V5 & 0.812 & accept & 1 & accept \\
\hline
\end{tabular}

It indicated that: For monthly IPO stock cumulative return, in 3 months, that is, in the window period $(0,60)$, the significance of ANOVA was greater than 0.05 , hence the null hypothesis was not rejected and the return under each sentiment level showed no significant differences. While, after a certain period, differences became significant since the fourth month or the window period $(60,100)$.

For monthly IPO stock cumulative volatility, in 5 months, that is, in the window period $(0,100)$, the volatility under each sentiment level showed no significant differences, that the impact of investor sentiment on IPO stock volatility was not obvious.

Multiple comparison by Scheffe and LSD two methods were also given to show the differences between each two group. For stocks listed for four months, cumulative return between each two investor sentiment levels almost appeared a significant difference, which verified the results above. 
Table 2 Multiple comparison of the monthly stock performance

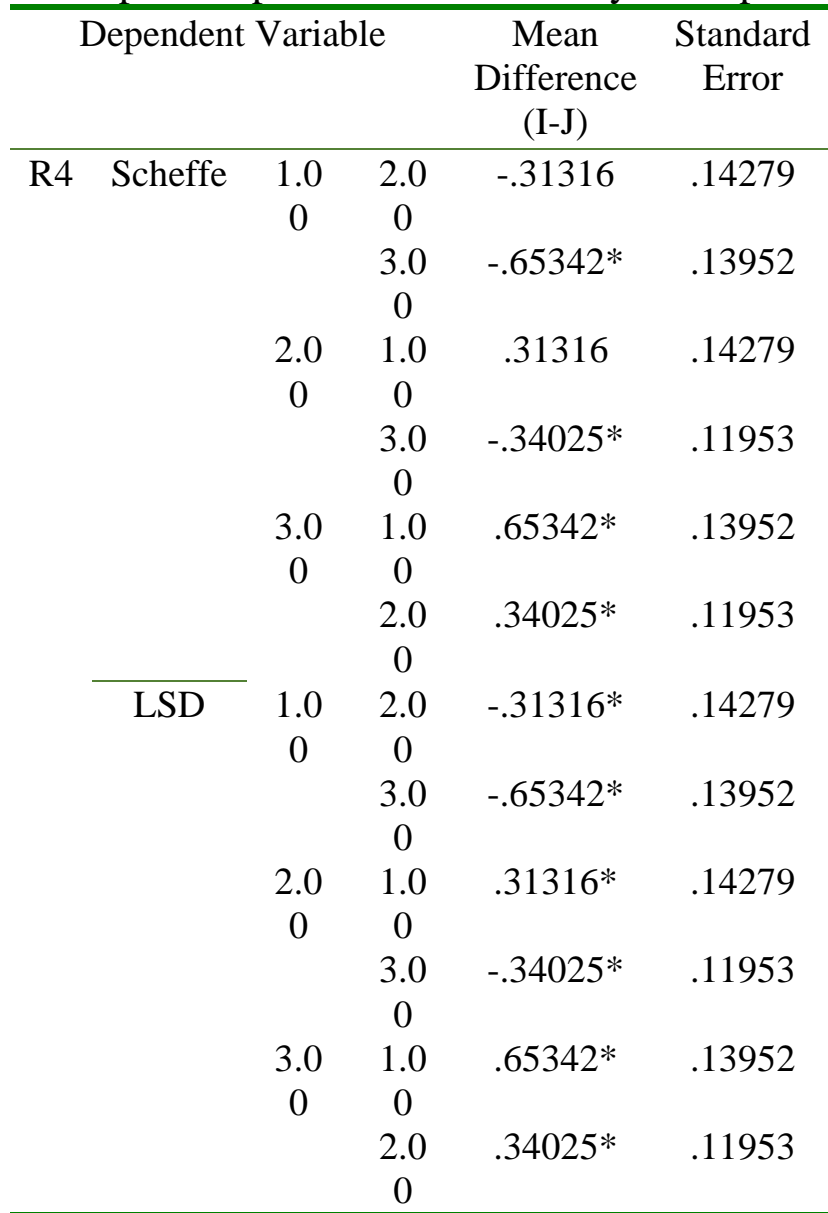

Afterwards, we implemented above tests and analysis on the daily stock data with same steps, whose results validated our findings. That is, the IPO stock return was not significantly affected by investor sentiment in the window period $(0,20),(20,40),(40,60)$. Take the result in $(0,20)$ as an example, it can be seen that ANOVA is not significant. In addition, as the market risk was taken into account, the significance of stock cumulative abnormal return is less than stock cumulative return.

Table 3 ANOVA of daily stock performance under different sentiment levels

\begin{tabular}{ccccc}
\hline \multicolumn{5}{c}{ Test of Homogeneity of Variance } \\
\hline \multirow{2}{*}{ R20 } & Levene & df1 & df2 & significance \\
& 2.200 & 2 & 687 & .111 \\
CAR20 & 1.204 & 2 & 7 & \\
& & & 7 & .300 \\
\hline
\end{tabular}

\begin{tabular}{ccccccc}
\hline \multicolumn{7}{c}{ ANOVA } \\
\hline & & Sum of squares & df & Mean & F & significance \\
R20 & between groups & .079 & 2 & .040 & 1.23 & .290 \\
& & & & & 7 & \\
CAR20 & between groups & .067 & 2 & .033 & 1.03 & .355 \\
& & & & & 7 & \\
\hline
\end{tabular}

\section{Summary}

The paper discussed how the IPO stock performance was significantly affected by investor sentiment, where a sentiment factor was proposed by cluster analysis towards three investor sentiment proxy indicators (turnover rate, number of new investors and CCI) and stock performance was represented 
by cumulative abnormal return, cumulative return and cumulative volatility. Both monthly data and daily data are considered in the study.

Conclusions are drawn that the IPO stock performance is basically not affected significantly by market sentiment in the window period $(0,60)$, for it is hard to predict the stock price without enough historical data, especially when information asymmetry and emotional release process existed in the market; however, after a certain period the market sentiment gradually shows obvious effect on IPO stock performance, manifested by gradually being independent of the IPO market and conforming to the general market since the fourth month or the window period $(60,100)$. In addition, the impact of market sentiment on stock return showed more significantly than that on volatility.

With more and more public information coming, fluctuations of IPO stock performance present a decreased tendency and differences between stocks become minor, in which process the influence of stocks weakened while the market impact enhanced.

\section{References}

[1] Lu P, Luo Q. Investor sentiment index construction - a new viewpoint on IPO initial return [J]. Journal of Shanxi University of Finance and Economics, 2010(3):55-61.

[2] Lo, A. W. and Wang, J., Trading Volume: Implications of an Intertemporal Capital Asset-Pricing Model [J]. Journal of Finance, 2006, 61(6), 2805-2840

[3] Wu Y, Han L. Imperfect rationality, sentiment and closed end fund puzzle [J].Economic research Journal, 2007(3):117-129.

[4] Jegadees and Titman, Returns to Buying Winners and Selling Losers: Implications for Stock Market Efficiency, 1993

[5] Cao Y. An investor composite sentiment index in China [D]. Harbin Institute of Technology, 2009.

[6] Huang X. Impact of investor sentiment on market expected return and volatility: An empirical study based on industry difference [D]. Fudan University, 2012.

[7] Shiller R.J. Irrational Exuberance [M]. Princeton University Press. 2005 